Pacific Journal of Mathematics

A GENERAL VERSION OF BELTRAMI'S THEOREM IN THE 


\title{
A GENERAL VERSION OF BELTRAMI'S THEOREM IN THE LARGE
}

\author{
H. Busemann and B. B. Phadke
}

\begin{abstract}
Beltrami's Theorem (1865) determines the Riemann spaces whose geodesics behave locally like affine lines. Its global form (proved much later) states that a complete simply connected Riemann space with this property is a euclidean, hyperbolic or spherical space. Here we establish what we believe to be the most general meaningful version of this theorem. We define a general class of complete metric spaces, called chord spaces, which possess distinguished extremals. In our case these must behave locally like affine lines, but they need not be the only extremals. This situation occurs in many important spaces.

Our principal result is that such a space (of $n$ dimensions, $n>1$ ) can be mapped topologically and geodesically either on the entire $n$-sphere $S^{n}$ or on an arbitrary open convex subset of an open hemisphere of $S^{n}$, considered as the affine space $A^{n}$. Examples of such spaces with some unexpected phenomena and the significance of chord spaces in general are discussed.
\end{abstract}

1. Introduction. The classical theorem of Beltrami was published in 1865 in [1]. It states that an $n$-dimensional Riemann space (Beltrami assumes $n=2$, but this case implies the result for $n>2$. We take $n>1$ throughout) in which the geodesics behave locally like projective lines has constant curvature and is therefore, according to Riemann, locally euclidean, hyperbolic or spherical.

The corresponding theorem in the large which was proved only in this century is: If the space $R$ is (in addition) complete and simply connected then it is a spherical space $S^{n}$ or is defined in an open hemisphere of $S^{n}$, i.e. the affine space $A^{n}$ and either $R=A^{n}$ is the euclidean space or, with a proper choice of the hemisphere $R$ is a hyperbolic space defined in the interior of an ellipsoid in $A^{n}$.

The proofs use strongly the properties of these three metrics.

The purpose of the present paper is to give a Beltrami's Theorem (B.T.) in the large the most general form which seems reasonable. Because the Theorem of Desargues plays a fundamental role we call for brevity a space locally desarguesian (1.d.) ${ }^{1}$ when each point has a neighborhood

\footnotetext{
${ }^{1}$ Our paper [9] treats special cases of interest and some independent topics. At the time we were not aware of the monodromy argument. We used it in a very similar form in [11]
} 
which can be mapped topologically and geodesically on a convex set $V$ in the projective space $P^{n}$, where convex means that there is a hyperplane $H \subset P^{n}$ with $V \cap H=\varnothing$ and $V$ is convex as set in the affine space $P^{n}-H$. Although it proves convenient to use a metric it turns out to be immaterial which model of $P^{n}$ or $S^{n}$ we use and we agree to always take $P^{n}$ or $S^{n}$ with curvature 1.

The usual setting for generalizations of the present type has been $G$-spaces, see [ 3 quoted as G]. But some of the most interesting and important desarguesian spaces are not $G$-spaces. [8], which is not readily accessible, gives axioms for a wider class of spaces where certain shortest joins, called chords, are distinguished. They comprise the 1.d. spaces where locally the projective lines furnish shortest connections but not necessarily the only ones. We give here a different, more concise, set of axioms for these "chord spaces" and discuss in $\S 2$ those of their properties which are needed here.

We split the B.T. in the large into two parts of which the first is of a topological nature and our main contribution. The types of simply connected spaces become very numerous for large $n$, so that it is not at all obvious that the very general metrics admitted in chord spaces do not lead to other image spaces than $S^{n}$. We prove, keeping in mind that chord spaces are by definition complete:

MAIn Theorem. A locally desarguesian simply connected chord space $R$ is either defined in all of $S^{n}$ or is an arbitrary open convex set of an open hemisphere of $S^{n}$ (considered as $A^{n}$ ).

That each convex subset $R$ of $A^{n}$ can be metrized as a straight $G$-space in infinitely many essentially different ways is contained in the proof of $\mathrm{G}(18.14)$. This is one reason why an analogue to the proof in the Riemannian case is not feasible.

Pogorelov [17] determined all globally desarguesian $G$-spaces. We will see that his results can be used to also determine all l.d. chord metrics so that we have the following

METRIC COMPLEMENT TO THE MAIN THEOREM. All simply connected locally desarguesian chord spaces are also known metrically.

The general 1.d. chord spaces are those covered by the simply connected ones. But they are not all known even in the Riemannian case, in particular our knowledge on the hyperbolic case is very lacunary. 
This material forms the content of $\S 3$. Finally we owe the reader cogent reasons which force us to go beyond $G$-spaces. In $\$ 4$ we provide examples showing the importance of admitting chord spaces. In addition it will become clear that chord spaces are interesting quite independently of their significance for desarguesian spaces.

2. Chord spaces. Our space $R$ is always a metric space with distance between $x$ and $y$ denoted simply by $x y$ satisfying the metric space axioms:

$$
\begin{aligned}
& x y \text { is real valued and }>0 \text { if } x \neq y, \\
& x x=0, \quad x y=y x \text { and } x y+y z \geq x z
\end{aligned}
$$

for any $x, y, z$ in $R$. Convergence of sets in $R$ is understood in terms of Hausdorff's closed limit, see $G \S 3$. When points $a, b, c$ satisfy $a b+b c=a c$ we say that $b$ lies between $a$ and $c$ and denote this by the symbol $(a b c)$; here $a, b, c$ are assumed to be distinct. Length of curves in $R$ is defined in the usual manner and a segment $T(a, b), a \neq b$, is an isometric map $x(t)$ of an interval of length $a b$ of the real $t$-axis $\mathbf{R}$ into $R$ i.e. $x(t)$ is defined in an interval $\alpha \leq t \leq \beta=\alpha+a b, x(\alpha)=a, x(\beta)=b$,

$$
x\left(t_{1}\right) x\left(t_{2}\right)=\left|t_{1}-t_{2}\right| \text {. }
$$

We note that if $a_{\nu} \rightarrow a, b_{\nu} \rightarrow b \neq a$ and if segments $T\left(a_{\nu}, b_{\nu}\right)$ exist and converge then the limit is a segment $T(a, b)$.

For a given subset $\overline{\mathscr{C}}$ of the set of all segments in $R$ a partial $\overline{\mathscr{C}}$-extremal is a class of maps $\{x(t), M\}$, where $M$ is a closed connected subset with more than one point of the reals $\mathbf{R}, t \in M, x(t) \in R$ and for each $t$ in $M$ numbers $t^{\prime}<t<t^{\prime \prime}$ exist with

$$
x\left(t_{1}\right) x\left(t_{2}\right)=\left|t_{1}-t_{2}\right| \text { for } t_{1}, t_{2} \text { in }\left[t^{\prime}, t^{\prime \prime}\right] \cap M
$$

and

$$
x(t) \mid\left[t^{\prime}, t^{\prime \prime}\right] \cap M \in \overline{\mathscr{C}} .
$$

In the cases $M=\mathbf{R}$ or $M=[\alpha, \beta]$ we use the terms $\overline{\mathscr{C}}$-extremal or $\overline{\mathscr{C}}$-extremal curve respectively. If $M=[\alpha, \beta]$ and $(*)$ and $(* *)$ hold for all

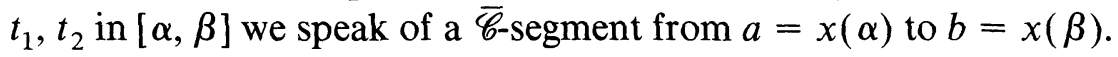

If the class is restricted to maps $t \rightarrow t+\eta$ we obtain an oriented partial $\overline{\mathscr{C}}$-extremal,..., oriented $\overline{\mathscr{C}}$-segment.

A $\overline{\mathscr{C}}$-extremal $\{x(t), \mathbf{R}\}$ is a $\overline{\mathscr{C}}$-straight line or simply a $\overline{\mathscr{C}}$-line if $(*)$ holds for any $t_{1}, t_{2}$. It is a $\overline{\mathscr{C}}$-great circle of length $\beta>0$ if

$$
x\left(t_{1}\right) x\left(t_{2}\right)=\min _{\nu=0, \pm 1, \pm 2, \ldots}\left|t_{1}-t_{2}+\nu \beta\right| .
$$


We now state the axioms for a space $R$ to be a chord space.

I. $R$ satisfies the conditions I, II, III of G-spaces which are (see G p. 37):

(i) The space is metric with distance $x y$.

(ii) The space is finitely compact, i.e. a bounded infinite set has at least one accumulation point.

(iii) Given two distinct points $x, z$ then a point $y$ with $(x y z)$ exists.

Before stating the second set II of axioms we note that it follows from I that any two points can be joined by a segment, this is proved in $G(6.8)$.

The second set of axioms is:

II. There is a set $\mathscr{C}$ of segments termed chords $Q(a, b)$. Calling $\mathscr{C}$-extremals and $\mathscr{C}$-extremal curves geodesics resp. geodesic curves we require:

(a) Each class $\mathscr{H}$ of homotopic curves form $a$ to $b$ (for given $a, b$ with $\mathscr{H} \neq \varnothing$ for $a=b$ ) contains a shortest curve which is a geodesic curve.

(b) The limit of a converging sequence $Q\left(a_{\nu}, b_{\nu}\right)$ with $a_{\nu} \rightarrow a, b_{\nu} \rightarrow b \neq$ a is $a Q(a, b)$.

(c) If two chords $Q(a, b)$ and $Q\left(a, b^{\prime}\right)$ with $a b \geq a b^{\prime}$ have a common point other than a then $Q\left(a, b^{\prime}\right) \subset Q(a, b)$ or $Q(a, b) \cap Q\left(a, b^{\prime}\right)=\{a, b\}$. (Trivially $b=b^{\prime}$ in the latter case.)

(d) Each point has a neighborhood $S\left(p, \sigma_{p}\right), 0<\sigma_{p}$, such that for $a, b$ in $S\left(p, \sigma_{p}\right)$ a $Q(a, c) \ni Q(a, b)$ exists.

The notations $A \Subset B$ or $B \ni A$ mean that $A$ is a proper subset of $B$.

We note some consequences of the axioms. From (a) alone (I is always assumed) we have

(1) $Q(a, b)$ exists for $a \neq b$. Every subarc of $Q(a, b)$ is a chord.

From (a) and (c)

(2) When $b^{\prime}$ is an interior point of $Q(a, b)$ then $Q\left(a, b^{\prime}\right)$ is unique and $a$ subarc of $Q(a, b)$.

(3) If $Q\left(a, c_{i}\right) \ni Q(a, b)$ and $b c_{1}=b c_{2}$ (or equivalently $\left.a c_{1}=a c_{2}\right)$ then $Q\left(a, c_{1}\right)=Q\left(a, c_{2}\right)$.

If not then (c) would imply $Q\left(a, c_{1}\right) \cap Q\left(a, c_{2}\right)=\left\{a, c_{1}\right\}$ but $Q(a, b) \subset Q\left(a, c_{i}\right), i=1,2$.

Let $\sigma(p), \sigma^{\prime}(p)$ be resp. the supremum of the $\rho$ for which (d) holds resp. $Q(a, b)$ is unique for $a \neq b$ in $S(p, \rho)$.

Since $Q(a, c) \equiv Q(a, b)$ implies by (2) that $Q(a, b)$ is unique we have first that $Q(a, b)$ is unique in $S\left(p, \sigma_{p}\right)$. Then we have using $\mathrm{G}(7.5)$ 
that $\sigma^{\prime}(p) \geq \sigma(p)>0$. (d) holds in $S(p, \sigma(p))$ and

(4) $\sigma(p) \equiv \infty$ or $0<\sigma(p)<\infty$ and $|\sigma(p)-\sigma(q)| \leq p q$.

The proof is similar to $\mathrm{G}(7.5)$ and the same proof shows that $\sigma^{\prime}$ satisfies the relations (4) too.

We note, without using it, that in our case $\sigma(p) \equiv \sigma^{\prime}(p)$ because a 1.d. space is a topological manifold, see [7, p. 19].

(5) If $p a<\sigma(p)$, a chord $Q(a, b) \ni p$ with $p a=p b$ exists.

The proof of (5) is given explicitly in $\mathrm{G}(7.8)$.

(6) $A$ chord can be extended uniquely to a geodesic. $\sigma(p) \equiv \infty$ characterizes the straight chord spaces, i.e. those where all geodesics are straight lines.

The proof is a simple combination of the arguments for $G(7.9)$ and $(8.4,5)$.

(7) If a geodesic contains with any $a \neq b a Q(a, b)$ then it is either $a$ straight line or a great circle.

The proof is identical with that of $\mathrm{G}(9.6)$. Although we always assume $\operatorname{dim} R>1$ we mention for justification the following corollary of (7) (whose proof is as in $\mathrm{G}(9.7)$ ).

(8) A 1-dimensional chord space is a straight line or a great circle.

REMARK. Dimension of a chord space is understood as its dimension as a metric space as defined, for example, in Hurewicz and Wallman [14].

The following Theorem (10) is known for $G$-spaces but not found in $\mathrm{G}$ or [7] but in [9, (2.8)]. Because it is clearly of prime importance for B.T. we provide a proof for chord spaces, in particular because it is partly superior to, and more complete than the argument in [9, (2.8)].

But first we note:

(9) Let $x_{\nu}(t), \nu=0,1,2, \ldots$, represent geodesics and $\lim _{\nu \rightarrow \infty} x_{\nu}(t)=$ $x_{0}(t)$ for $t \in[\alpha, \beta], \alpha \neq \beta$. Then $x_{\nu}(t)$ converges to $x_{0}(t)$ uniformly on any bounded set of $\mathbf{R}$.

The proof is like that of $\mathrm{G}(8.11,12)$.

(10) THEOREM. If an open set $R \neq \varnothing$ of $S^{n}$ is metrized as a chord space whose geodesics fall on the great circles in $S^{n}$ then $R$ is either a convex set in an open hemisphere (i.e. $A^{n}$ ) and straight or $R=S^{n}$ and $S^{n}$-antipodes are $R$-antipodes.

When $a \neq b$ are points of $R$ and not antipodes in $S^{n}$, then the great circle on $S^{n}$ and hence the $R$-geodesic through $a$ and $b$ is unique. It 
contains all $Q(a, b)$ because each lies on a geodesic. When $a, b$ are antipodes, choose a $Q(a, b)$ and a sequence $b_{\nu} \rightarrow b, b_{\nu} \neq b, b_{\nu} \in Q(a, b)$. Then $a$ and $b_{\nu}$ are not antipodes hence $Q\left(a, b_{\nu}\right) \subset Q(a, b) \subset R$ and (9) shows that there is a geodesic containing $Q(a, b)$; moreover $Q(a, b)$ is an $S^{n}$-semigreat circle. It follows from (7) that each geodesic in $R$ is a great circle or a straight line.

A chord in $R$ which is a semigreat circle $Q(a, b)$ cannot be prolonged to a chord $Q(a, c) \ni Q(a, b)$. For if $d$ is an interior point of the subchord $Q(b, c)$ then both arcs from $a$ to $d$ of the $S^{n}$-great circle containing $Q(a, b)$ would be a $Q(a, d)$ and unique, one because $a$ and $d$ are not antipodes, the other because a proper subchord of a chord is unique.

To finish the proof we show: if $R$ contains two antipodes $a, b$ then $R=S^{n}$. We proved that with any other point $q$ of $R$ the entire $S^{n}$-great circle through $a, b, q$ lies in $R$. For an arbitrary $p(\neq a, b)$ in $S^{n}$ the $S^{n}$-great circle $C^{\prime}$ through $a, b, p$ contains other points of $R$ because a neighborhood of $a$ lies in (the open) $R$. Therefore $p \in C^{\prime} \subset R$.

If in our $S^{n}$ as given chord space we identify antipodes we obtain a chord metric in $P^{n}$, see G p. 129.

We will see in $\$ 4$ that often fundamentally different chord spaces may be constructed with the same metric. But if for some two points $a \neq b$ the segment $T(a, b)$ is unique, then it must be a $Q(a, b)$ in any compatible chord space. This implies that if the given metric defines a $G$-space then the only chord space compatible with it is the space with all $T(a, b)$ as chords. For $(a b c)$ in a $G$-space the segment $T(a, b)$ is unique, so that the $Q(a, b)$ are determined locally.

Therefore

(11) $A$ chord space is a G-space iff all segments are chords. In this case the metric determines the chord space uniquely.

The same argument shows

(12) If a space is metrized such that $T(a, b)$ always exists and is unique, then the space is either a chord space with the $T(a, b)$ as $Q(a, b)$ or there is no compatible chord space.

The latter may happen even when for given $a \neq b$ and $\rho>0$ a $c$ with $b a=\rho$ and $(a b c)$ exists. For example, consider the following metrization of the $\left(x^{1}, x^{2}\right)$-plane. For $x=\left(x^{1}, x^{2}\right)$ and $y=\left(y^{1}, y^{2}\right)$ we define

$$
\begin{aligned}
& x y=\sqrt{\left(x^{1}-y^{1}\right)^{2}+\left(x^{2}-y^{2}\right)^{2}}+\left|x^{1}-y^{1}\right| \quad \text { if } x^{2}, y^{2} \geq 0 \\
& x y=2 \sqrt{\left(x^{1}-y^{1}\right)^{2}+\left(x^{2}-y^{2}\right)^{2}} \quad \text { if } x^{2}, y^{2} \leq 0
\end{aligned}
$$




$$
x y=\min _{t}(x(t, 0)+(t, 0) y) \quad \text { if } x^{2} \leq 0 \text { and } y^{2} \geq 0 .
$$

Then the prolongation of, for example, the segment along the $x^{2}$-axis from $a=(0,1)$ to $z=(0,0)$ is not unique. None of these many continuations of $T(a, z)$ can be omitted because otherwise no $T(a, c)$ for some $c=\left(c^{1}, c^{2}\right)$ with $c^{2}<0$ would exist.

(For suitable conditions which prevent this nonuniqueness see [12] where we construct a class of examples of $G$-spaces).

3. Locally desarguesian spaces. In this paper we call a subset $Q$ of a chord space $R$ "convex" if the following condition holds: when $x$ and $y$ lie on $Q$ there is one and only one chord $Q(x, y)$ in $R$ joining $x$ and $y$ and this chord is contained in $Q$.

When $R$ is the euclidean space, this concept of convexity coincides with the usual affine space convexity. We note also that our definition of convexity above yields the following notion of convexity in the projective space $P^{n}$ : a set $Q$ in the real projective space $P^{n}$ is called convex if $Q$ is convex in the $G$-space sense above when $P^{n}$ is metrized as the elliptic space. (The elliptic space is a $G$-space). This notion of convexity, of course, coincides with the definition in the Introduction. Finally we mention the case when $R$ is the spherical space $S^{n}$. Here our definition amounts to the following: $Q \subset S^{n}$ is convex if $Q$ lies in an open hemisphere and if for $x, y$ in $Q$ the shorter of the two arcs of the great circle containing $x$ and $y$ lies in $Q$.

We now define and explain the notion of a locally desarguesian chord space. A chord space $R$ is said to be locally desarguesian if each point $p$ of $R$ is contained in a nonempty open convex set $U$ which can be mapped homeomorphically onto an open convex set $\tilde{U}$ in the real affine space $A^{n}$ in such a way that the geodesic curves contained in $U$ are mapped onto affine segments in $\tilde{U}$. (Such a map is called a geodesic map.) This is equivalent to saying that each point $p$ of $R$ is contained in an open convex set which can be mapped homeomorphically and geodesically onto an open convex set of the sphere $S^{n}$, or the projective space $P^{n}$.

In the sequel we use throughout a fixed sphere $S^{n}$ (and neither $P^{n}$ nor $\left.A^{n}\right)$ as the "model space" i.e. the space into which we map.

If the open convex set $U$ of $R$ is mapped homeomorphically and geodesically onto an open convex subset of $S^{n}$ by means of a map $\phi$ we say that $U$ is a "desarguesian domain" and $\phi$ is a "desarguesian chart". The pair $(U, \phi)$ is called a "desarguesian element".

The connection between our use of the word "desarguesian" and the theorem of Desargues derives from the "Desargues Property" formulated 
for $G$-spaces by $\mathrm{H}$. Busemann and the implications of this property for $G$-spaces; see [G, Chapter II, §§12, 13 and 14].

The arguments in the proof of our Main Theorem are to a great extent simplified because the following properties hold for our desarguesian domains and charts:

(A) Standardization of the size of a domain: There exists a positive continuous function $\gamma(p)$ such that each closed ball $\bar{S}(p, \gamma(p))$ lies in a desarguesian domain $W(p) \ni p$.

(B) Uniqueness of charts: If $(U, \phi)$ and $(V, \psi)$ are two desarguesian elements and $\phi=\psi$ on a nonempty open set $Z \subset U \cap V$ then $\phi=\psi$ on the entire intersection $U \cap V$.

(C) Freedom of transition from one chart to another: If $(W, \psi)$ and $(Z, \phi)$ are desarguesian elements and $W \supset Z$ then there exists a desarguesian element $(W, \Phi)$ such that $\Phi \mid Z=\phi$ i.e. the restriction of $\Phi$ to $Z$ coincides with $\phi$.

We now establish these properties. First consider (A). The proof of this uniformity of standardized sizes is based on the frequently used idea of [G (7.5) p. 33]. In fact, let $\mu(p)$ be the supremum of positive numbers $\alpha$ such that $S(p, \alpha)$ is contained in some desarguesian domain $V$. Since $R$ is locally desarguesian, $\mu(p)$ is positive. When $S(p, \alpha)$ is contained in $V$, $S(q, \alpha-p q)$ is also contained in $V$ if $\alpha>p q$. Therefore, $\mu(p)$ is either identically equal to $\infty$ or else $|\mu(p)-\mu(q)| \leq p q$ for all $p, q$ in $R$. Hence if we put, for example, $\gamma(p)=\frac{1}{2} \min (\mu(p), \sigma(p))$, then each closed $\bar{S}(p, \gamma(p))$ is contained in some desarguesian domain $W(p)$. We note that since $\gamma(p)$ is continuous and positive, it is bounded away from zero on any compact set.

We now consider properties (B) and (C). The proof of these properties is, in turn, based on the following facts about geodesic maps of the sphere $S^{n}$ onto itself. (Such bijective homeomorphisms of $S^{n}$ onto $S^{n}$ sending great circles to great circles are also called spherical collineations.)

I. A spherical collineation sends a convex set onto a convex set.

II. If $f$ and $g$ are spherical collineations and if $f=g$ on a nonempty open set $U$ then $f=g$ on the whole of $S^{n}$.

III. Let $\tilde{f}$ be a bijection of a nonempty open convex set $Q \subset S^{n}$ onto a nonempty open convex set $Q^{\prime} \subset S^{n}$ such that $\tilde{f}$ sends arcs of great circles in $Q$ onto arcs of great circles in $Q^{\prime}$. Then $\tilde{f}$ can be extended to a collineation $f$ of $S^{n}$. The extension $f$ of $\tilde{f}$ is unique.

The proofs of these three properties are fairly standard and can be obtained by using information about projective collineations and the standard covering of $P^{n}$ by $S^{n}$. 
We now derive the properties (B) and (C).

For (B) we simply have to observe that if $\tilde{U}=\phi(U)$ and $\tilde{V}=\psi(V)$ and $\tilde{Z}=\phi(Z)=\psi(Z)$ then $\phi \circ \psi^{-1}$ sends $\tilde{Z}$ to $\tilde{Z}$ and is the identity on $\tilde{Z}$ and hence its unique extension to $S^{n}$ is the identity. Hence $\phi \circ \psi^{-1}$ is the identity on $\psi(U \cap V)$ also. Hence $\psi(U \cap V)=\phi(U \cap V)$ and $\phi=\psi$ on $U \cap V$.

Finally we consider (C). Here the map $\tilde{g}=\psi \circ \phi^{-1}$ from $\phi(Z)$ to $\psi(Z)$ satisfies the hypothesis in III above and hence has an extension $g$ to $S^{n}$. We now define $\Phi=g^{-1} \circ \psi$ so that $\Phi \mid Z=\phi$.

From now on we assume that all our desarguesian domains have the property that if $U$ is a desarguesian domain then there exists at least one $p \in U$ such that $\bar{S}(p, \gamma(p))$ is contained in $U$.

When desarguesian elements $(U, \psi)$ and $(V, \phi)$ satisfy the condition that $\psi=\phi$ on $U \cap V$ and $U \cap V$ is nonempty we say that $(U, \psi)$ and $(V, \phi)$ are immediate continuations of each other.

Let $(U, \phi)$ be a desarguesian element and let $C$ be a continuous curve parametrized by $\alpha(t), a \leq t \leq b$, with $\alpha(a) \in U$. We say that $(U, \phi)$ is continued along $C$ to $(V, \psi)$ if there exists a partition $\Delta: a=t_{0}<t_{1}<t_{2}$ $<t_{3}<\cdots<t_{k}=b$ and a family $\left(U_{i}, \phi_{i}\right), i=0,1,2, \ldots, k$, of desarguesian elements such that:

(i) $\alpha\left(\left[t_{l}, t_{i+1}\right]\right) \subset S\left(\alpha_{i}, \gamma_{\alpha} / 2\right) \subset \bar{S}\left(\alpha_{i}, \gamma_{\alpha} / 2\right) \subset U_{i}$ where $\alpha_{i}=\alpha\left(t_{i}\right)$ and $\gamma_{\alpha}=\inf \{\gamma(\alpha(t)), a \leq t \leq b\}>0 .\left(\gamma_{\alpha}>0\right.$ since $\gamma$ is continuous and the trace of, i.e. the actual pointset occupied by, $\alpha(t)$ is a compact set).

(ii) $\left(U_{i}, \phi_{i}\right)$ and $\left(U_{i+1}, \phi_{i+1}\right)$ are immediate continuations of each other, $i=0,1, \ldots, k-1$.

(iii) $U \subset U_{0}, \phi_{0}=\phi$ on $U, V \subset U_{k}$ and $\psi=\phi_{k}$ on $V$.

If a curve $C$ joins $p$ to $q$ and lies completely in a single desarguesian domain $W$ then from properties (B) and (C) we conclude that continuation of any element $(U, \phi), p \in U$ along $C$ to $(V, \psi)$ exists; in fact we can take $V=W$ and $\phi=\psi$ on $W \cap U$.

Since any general continuous curve $\alpha(t), a \leq t \leq b$, has a compact trace which can be covered by the union of a finite number of desarguesian domains of appropriate sizes satisfying condition (i) above we conclude that continuations of desarguesian elements along all curves exist.

We now show that continuation is "unique". First consider the case when $C_{1}$ and $C_{2}$ are two curves both joining $p$ to $q\left(C_{1}=C_{2}\right.$ is not excluded) and both lying in a single desarguesian domain $W$. Then if $\left(U_{i}, \phi_{i}\right)$ are continuations of $(U, \phi)$ along $C_{i}, i=1,2$, then the properties (B) and (C) above show that $\phi_{1}=\phi_{2}$ on $U_{1} \cap U_{2}$. Thus, in single desarguesian domains continuation is independent of the path. In other 
words a single desarguesian domain $W$ is always a "neutral" neighborhood for continuation. (Compare arguments on p. 43 of [11].)

We remember now that our chord space $R$ is simply connected and consider the case when $C_{1}$ and $C_{2}$ are any two curves both joining $p$ to $q$. ( $C_{1}=C_{2}$ is not excluded.) Then since $C_{1}$ and $C_{2}$ are homotopic we can subdivide the deformation square of the deformation homotopy into a finite number of small subsquares in such a way that the image of each subsquare lies in a "neutral" neighborhood. A familiar argument (similar to those occurring in some proofs of the monodromy theorem, for example, in complex analysis) then shows that if $(U, \phi)$ is continued to $\left(U_{i}, \phi_{i}\right)$ along $C_{i}, i=1,2$, then $\phi_{1}=\phi_{2}$ on $U_{1} \cap U_{2}$. (Again compare [11, pp. 43, 44].)

Thus we have:

LEMMA. Let $R$ be a simply connected locally desarguesian chord space and let $(U, \phi)$ be a desarguesian element. Then $(U, \phi)$ can be continued along all curves beginning in $U$. If $C_{1}$ and $C_{2}$ are two curves starting at $p$ and ending at $q$ and if $\left(V_{i}, \phi_{i}\right), i=1,2$, are continuations of $(U, \phi)$ along $C_{i}$, $i=1,2$, respectively, then $\phi_{1}=\phi_{2}$ in the nonempty neighborhood $V_{1} \cap V_{2}$ of $q$.

Choose now a fixed point $z$ of $R$ and a fixed desarguesian element $(U, \phi)$ with $z \in U$. For an arbitrary point $x \in R$ let $C$ be a curve from $z$ to $x$ and $\left(U_{C}, \phi_{C}\right)$ be a desarguesian element obtained by continuing $(U, \phi)$ along $C$. Then from the lemma above we see that $x^{\prime}=\phi_{C} x$ is independent of the choice of the curve $C$ and the partition used during continuation. Hence the association of $x^{\prime}$ with $x$ yields a desarguesian chart in the neighborhood $W(x)$ of $x$. We denote by $\Delta$ the map which sends $x \in R$ to $x^{\prime} \in S^{n}$ as above.

Since $\Delta$ is a desarguesian chart on each $W(x)$, the image under $\Delta$ of any geodesic in $R$ lies on (i.e. is contained in) a great circle in $S^{n}$. Using this and the local injectivity of $\Delta$ we show that $\Delta$ is actually globally injective i.e. if $a \neq b$ then $\Delta a \neq \Delta b$.

For an indirect proof assume that $\Delta a=\Delta b$ and $a \neq b$. Let $Q$ be any chord joining $a$ to $b$. Then $\Delta$ maps $Q$ onto a great circle $\Gamma$, say, in $R^{\prime}=\Delta R \subset S^{n}$. Hence on $Q$ there exist points $p, q$ such that $p^{\prime}=\Delta p$, $q^{\prime}=\Delta q ; p, q$ are distinct from $a, b$ and $p^{\prime}, q^{\prime}$ are antipodal on $\Gamma$. Since $R^{\prime}=\Delta R$ is an open subset of $S^{n}$ we can find another great circle $\Sigma \neq \Gamma$, $\Sigma \subset R^{\prime}, p^{\prime}, q^{\prime} \in \Sigma$. Choose now sequences $p_{n}^{\prime}, q_{n}^{\prime}$ on $\Sigma$ such that $p_{n}^{\prime}$ is not antipodal to $q_{n}^{\prime}, p_{n}^{\prime} \rightarrow p^{\prime}, q_{n}^{\prime} \rightarrow q^{\prime}$ as $n \rightarrow \infty$. As $\Delta$ is injective in neighborhoods of $p, q$ we can find sequences $p_{n}, q_{n}$ in $R$ such that $p_{n} \rightarrow p, q_{n} \rightarrow q$ 

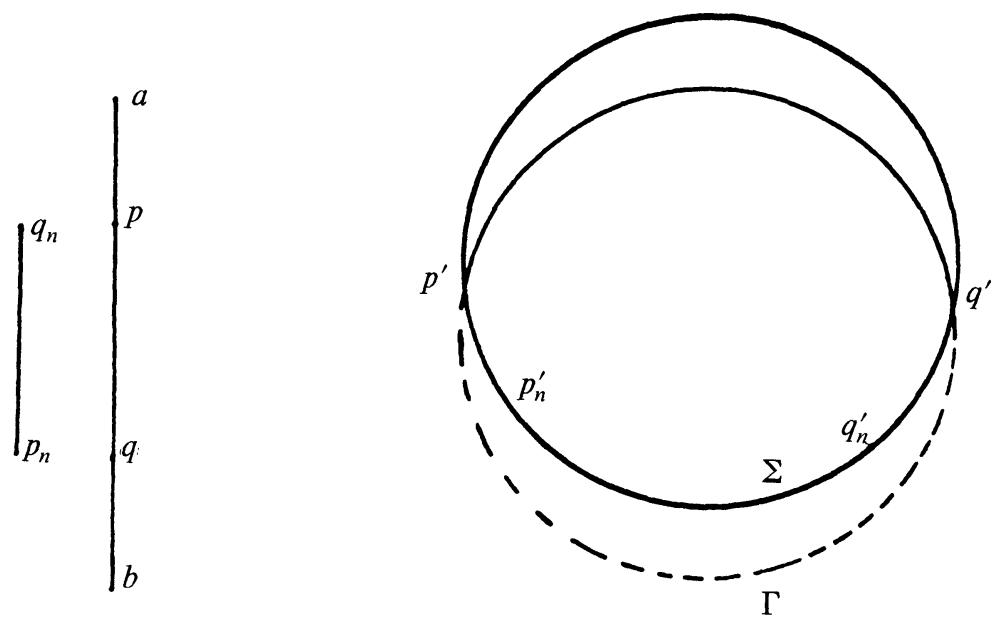

FigURe 1

and $\Delta p_{n}=p_{n}^{\prime}, \Delta q_{n}=q_{n}^{\prime}$. Let $Q_{n}$ be any chord joining $p_{n}$ and $q_{n}$. See Figure 1.

$Q(p, q)$ is unique since $p, q \in Q-\{a, b\}$, hence there is a subsequence, which we may again denote by $Q_{n}$ such that $Q_{n} \rightarrow Q_{0}=Q(p, q)$ $\subset Q$ as $n \rightarrow \infty$. Now $p_{n}^{\prime}, q_{n}^{\prime}$ are not antipodal, hence the great circle joining $p_{n}^{\prime}, q_{n}^{\prime}$ is unique, namely $\Sigma$. Hence $\Delta Q_{n} \subset \Sigma$ for all $n$. Therefore $\Delta Q_{0}=\Delta \lim Q_{n}=\lim \Delta Q_{n} \subset \Sigma$. Thus $\Delta Q_{0} \subset \Gamma \cap \Sigma$. This is a contradiction to $\Gamma \neq \Sigma$ and hence if $a \neq b$ then $\Delta a \neq \Delta b$ i.e. $\Delta$ is globally injective.

We have thus proved that $\Delta$ maps $R$ homeomorphically onto an open subset $R^{\prime}$ of $S^{n}$ in such a way that $\Delta$ sends the geodesics of $R$ onto portions of great circles of $S^{n}$. We may thus identify $R$ with $R^{\prime}$ and regard the latter as a chord space. Hence we can apply Theorem (10) of $\S 2$ to conclude that either $R^{\prime}$ lies in an open hemisphere of $S^{n}$ (i.e. $A^{n}$ ) and is a straight desarguesian chord space or else $R^{\prime}$ is the entire $S^{n}$ and antipodes in $R^{\prime}$ and $S^{n}$ coincide. This completes the proof of the Main Theorem as stated in the Introduction.

Pogorelov [17] determined all globally desarguesian (g.d.) $G$-spaces (in $\left.P^{n}\right)$. This allows us to construct all chord metrics in the sets occurring in the Main Theorem. In the case $R \subset A^{n}$ our spaces are g.d. If $x y$ metrizes $R$ as a g.d. chord space and $\varepsilon(x, y)$ is a Euclidean metric in $A^{n}$ (invariant under translations), then $R$ is with $x y+(1 / k) \varepsilon(x, y)$ a $G$-space. Because of Theorem (10) chords in $R$ and segments on $S^{n}$ coincide (as point sets) so that if $\sigma(x, y)$ is the spherical metric $x y+(1 / k) \sigma(x, y)$ is a $G$-metric on $S^{n}$ with the great circles as geodesics. 
Pogorelov's procedure is the same in both cases, so let $R \subset A^{n}$. He proves that each g.d. metric in $R$ can be approximated by explicitly given g.d. metrics $\rho_{\nu}(x, y)$ of class $C^{\infty}$, uniformly on any compact set. Applying this to $x y+(1 / k) \varepsilon(x, y)$ we find that any g.d. chord metric can be approximated uniformly on compact sets by definite $\rho_{\nu}(x, y)$ of class $C^{\infty}$. Evidently the limit of such $\rho_{\nu}$ is the given $x y$, thus all chord metrics can be constructed.

The result can be formulated in a more satisfactory way, because the $\rho_{\nu}(x, y)$ can evidently be varied. If $K_{M}$ is the set of all hyperplanes intersecting the point set $M$, then $\rho_{\nu}(x, y)$ can be written in the form

$$
\rho_{\nu}(x, y)=\mu_{\nu}\left(K_{T(x, y)}\right)=\int_{K_{T(x, y)}} f_{\nu}(H) d H
$$

where $f_{\nu}$ is smooth and $d H$ is the euclidean density of hyperplanes. For $\nu \rightarrow \infty$ we obtain a set function $\mu$ with the property that

$$
x y=\mu\left(K_{T(x, y)}\right) .
$$

Pogorelov determines for $G$-metrics precisely the $\mu$ with this property (he considers only $n=2,3$, but his method for $n=3$, where $\mu$ taking negative values must be admitted, works for $n>3$ ). We call these set functions briefly $P$-functions. If $W_{R}$ is the totality of all $P$-functions for $R$ and $W_{R}^{n}$ the subset of $W_{R}$ corresponding to the $G$-metrics of the form $x y+\delta \varepsilon(x, y), 0<\delta \leq 1 / n$, then $W_{R}^{n} \supset W_{R}^{n+1}$ and $\cap W_{n}=W$ contains all g.d. $G$-metrics in $R$ and in addition precisely the g.d. chord metrics.

That a metric analogue to the B.T. singling out three basic geometrics cannot exist without additional conditions is evident from our preceding considerations. All that can be expected is a condition weaker than constant curvature and meaningful in $G$-spaces. The following version may be of interest.

THEOREM. A simply connected locally desarguesian and locally symmetric G-space is Minkowskian, hyperbolic or spherical.

We indicate the proof briefly. Locally symmetric $G$-spaces which generalize locally symmetric Riemann and Finsler spaces are defined, see [11], as $G$-spaces in which a positive continuous $\beta(p)$ exists such that each $S(p, \beta(p))$ is symmetric in $p$. In [10, (4.2), (4.4)] we proved that a locally symmetric globally desarguesian $G$-space is Minkowskian or hyperbolic and that a locally symmetric spherelike $G$-space is spherical. These results when combined with the Main Theorem give the theorem stated above. 
4. The significance of chord spaces. Taking axioms I to IV of $G$-spaces we obtain extremals, but they may behave so pathologically that no theory of truly geometric interest can be built on these axioms alone. (We note here that the axioms I, II and III of $G$-spaces were stated in \$2 above. The axiom IV, see $\mathrm{G}$ p. 37, states: To every point $p$ of the space there corresponds a positive $\rho_{p}$, such that for any two distinct points $x, y$ in $S\left(p, \rho_{p}\right)$ (i.e. $\left.x p<\rho_{p}, y p<\rho_{p}\right)$ a point $z$ with $(x y z)$ exists. The axiom IId of chord spaces is an analogue to this axiom).

It is shown in [7, p. 7] that there are metrics in the plane where each segment can be continued to a straight line and which permit nevertheless closed extremals and also $x(t)$ defined in $(-\infty, \alpha), \alpha$ finite, satisfying (1) locally, but which cannot be continued to extremals. In [6] one finds for any plane Minkowski metric whose circles are polygons extremals which are transitive in a very strong sense.

All this holds for one of the most important Minkowski planes, namely those whose circles are parallelograms (which are all isometric by $\mathrm{G}(17.10)$ ). We denote this metric by $m_{p}$. In addition to $m_{p}$ there is another very important plane Minkowski metric $m_{h}$ whose circles are affine images of euclidean regular hexagons.

These metrics occur as solutions of various extremum problems of which we will discuss a few. They are all found in Petty [16]. The very simplest problem is: which are the plane Minkowski geometries yielding the longest or the shortest unit circle? The answer is that $m_{p}$ (and only $m_{p}$ ) yields the maximum 8 , similarly only $m_{h}$ yields the minimum 6.

Let $A, B$ be affine lines in $A^{2}$ intersecting at $p, A^{\prime}, B^{\prime}$ subrays of $A, B$ with origin $p$ and $A^{\prime \prime}, B^{\prime \prime}$ the opposite rays. A Minkowski metric is invariant under translations and the theory of Haar measure tells us that up to a factor only one measure invariant under translation exists which we normalize for each Minkowski metric such that the unit disk has measure $\pi$. If $a^{\prime} \in A^{\prime}-p, b^{\prime} \in B^{\prime}-p$ we define the Minkowski sine of the angle formed by $A^{\prime}$ and $B^{\prime}$ by

$$
\operatorname{sm}\left(A^{\prime}, B^{\prime}\right)=\frac{2 \Delta}{\sqrt{p a^{\prime} \cdot p b^{\prime}}}
$$

where $\Delta$ is the area of the triangle $p a^{\prime} b^{\prime}$. This is independent of the choice of $a^{\prime}, b^{\prime}$ and trivially $\operatorname{sm}\left(A^{\prime \prime}, B^{\prime \prime}\right)=\operatorname{sm}\left(A^{\prime}, B^{\prime}\right)$. But corresponding to $\sin \alpha=\sin (\pi-\alpha)$ we also have

$$
\operatorname{sm}\left(A^{\prime}, B^{\prime \prime}\right)=\operatorname{sm}\left(A^{\prime \prime}, B^{\prime}\right)=\operatorname{sm}\left(A^{\prime}, B^{\prime}\right) .
$$


Thus sm depends only on $A, B$ and we write $\operatorname{sm}(A, B)$. This can, in general, not be interpreted as a function of a number, but is evidently invariant under translation of $A$ or $B$, which we can therefore assume to pass through a fixed $p$.

If $B$ is kept fixed and parallel to the direction $\omega$ at $p$ then $A$ is perpendicular to $B$ or $B$ transversal to $A$ iff

$$
\operatorname{sm}\left(A^{\prime}, B\right) \leq \operatorname{sm}(A, B)
$$

for any $A^{\prime} \ni p$. We set $\alpha(B)=\operatorname{sm}(A, B)=\alpha(\omega)=\alpha(\omega+\pi)$. The function $\alpha$ plays an important role, for example in the solution of the isoperimetric problem, but that does not interest us here.

All metrics satisfy

$$
\pi / 4 \leq \alpha(\omega) \leq \pi / 2
$$

in this case both bounds are attained only by $m_{p}$. Also always

$$
\max \alpha(\omega) \geq 1,
$$

with equality only for the ellipse, i.e. the euclidean geometry. But $\min \alpha \leq$ $\pi / 3$ with the equality only for $m_{h}$.

The geometries for which perpendicularity is symmetric are those whose circles are Radon curves and they are characterized by $\alpha(\omega)=$ constant. The minimum and maximum for constants $\alpha$ are 1 and $\pi / 3$ because both in the euclidean metric and $m_{h}$ perpendicularity is symmetric.

As an example of a different type we consider the Hilbert geometries (see G $\$ 18$ and Hilbert [13, Appendix 1]). Let $S$ be a closed convex hypersurface in $A^{n}$ and $\mathscr{I}$ its interior. In $\mathscr{I}$ we define distance just as in hyperbolic geometry. If the affine line through $a \neq b$ in $\mathscr{I}$ intersects $S$ in $u$ and $v$, the distance

$$
h(a, b)=|\log \rho(a, b, u, v)|
$$

where $\rho$ is cross ratio. Hilbert shows that the triangle inequality holds and that the intersections of $\mathscr{I}$ with affine lines are straight extermals and the only ones (so that $\mathscr{I}$ becomes a $G$-space) iff no 2-plane intersecting $\mathscr{I}$ intersects $S$ in a curve containing two non-collinear affine segments. Taking affine segments in $\mathscr{I}$ as chords yields therefore a desarguesian chord space.

The most degenerate case is where $S \cup \mathscr{I}$ is a simplex. For $n=2$ this geometry and the hyperbolic (where $S$ is an ellipse) are the only ones with symmetric perpendicularity [15]. 
The case of general $n$ is most interesting. The (unique) Hilbert geometry possessing a transitive abelian group of motions where the affine segments are the chords (motion means that both distance and chords are preserved) is given by a simplex $S$, see [5, p. 35]. If we realize $\mathscr{I}$ as the first quadrant $x_{i}>0$ of an affine coordinate system, the group is given by $x_{l}^{\prime}=\beta_{i} x_{i}, \beta_{i}>0$.

Denoting by $\phi$ the map $y_{i}=\log x_{i}$ of $\mathscr{I}_{\text {on }} A^{n}$ the group becomes the group of translations of $A^{n}$ and putting $m\left(y^{\prime}, y^{\prime \prime}\right)=m\left(\phi x^{\prime}, \phi x^{\prime \prime}\right)=$ $h\left(x^{\prime}, x^{\prime \prime}\right)$ we get a chord-metric in $A^{n}$ invariant under the translations, whose chords are the images of the affine segments in $\mathscr{I}$. The geodesics are straight and satisfy the hyperbolic parallel axiom, moreover the Theorem of Desargues. $m$ is a Minkowski metric because it is invariant under the translations and we can take the affine segments as chords.

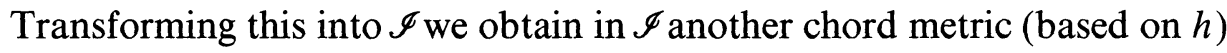
which satisfies both the parallel axiom and Theorem of Desargues.

From a general point of view we arrive at the very interesting question: If $R$ is a metric space satisfying axioms I, II, III and $\mathscr{C}(R)$ is the set of all chord spaces compatible with the given metric, how different can the elements of $\mathscr{C}(R)$ be? For example $\mathscr{C}\left(m_{p}\right)$ contains straight chord spaces whose geodesics (but not the metric) are invariant under the translations where the theory of parallels (into which we do not want to enter here) has none of the usual properties of euclidean or hyperbolic geometry. The examples given in G p. 140 confirm this.

This leads to the further question whether special metrics like the Minkowski metrics in $A^{n}$ can be characterized by intrinsic properties instead of defining the chords. In general this is clearly impossible owing to the arbitrariness in defining chords, but easy in the case mentioned.

A theorem of Pontrjagin [18, p. 170] states that a simply connected topological manifold which is the group space of a transitive abelian group of motions is equivalent to $A^{n}$ with the translations as group. The affine lines are the orbits of the one-parameter subgroups. This contains as special case that among the chord metrics in $A^{n}$ with the translations as motions the Minkowskian are those whose geodesics are the orbits of $A^{n}$ considered as group space. Applied to our example it determines in the Hilbert simplex at once the geodesics of the desarguesian chord metric with the parallel axiom as the orbits of $x_{i}^{\prime}=\beta_{i} x_{i}, 1 \leq i \leq n$.

We hope to have convinced the reader that a generalization of $G$-spaces is necessary and possible to obtain a complete analogy to the topological aspect of B.T. i.e. our Main Theorem, and that chord spaces 
are one possibility which leads in addition to many interesting new problems and often to unexpected phenomena.

Surprisingly, one can clearly deduce from a paragraph (which is too long to be produced here in toto) in Beltrami's paper [2] that he would have welcomed our generalization of his theorem. He says that a theorem which holds under weaker hypotheses than stated has not been fully understood and that the proper generalization may involve the disappearance of some of the original concepts (in our case the Riemannian metric).

\section{REFERENCES}

[1] E. Beltrami, Risoluzione del problema: riportare i punti di una superficie sopra un piano in modo che le linee geodetiche vengano rappresentate da linee rette, Opere, I (1865), 262-280.

[2] __ Saggio di interpretazione della Geometria non-Euclidea, Giornale di Matematiche, VI (1868), 284-312.

[3] H. Busemann, The Geometry of Geodesics, (1955), Academic Press, New York.

[4] __ Geometries in which the planes minimize area, Ann. Mat. Pura. Appl. (IV), 55 (1961), 171-190.

[5] Timelike spaces, Dissert. Math. No. 53, (1967), Warsaw.

[6] _. Transitive geodesics in Minkowski planes, Math. Chronicle, 1 (1969), 27-29.

[7] _ Recent Synthetic Differential Geometry, Ergeb. Math. Band 54, (1970), Springer-Verlag, New York.

[8] _ Spaces with Distinguished Shortest Joins, A Spectrum of Mathematics (essays presented to H. G. Forder, edited by J. C. Butcher; University Press, Auckland, New Zealand; Oxford University Press), (1971), 108-120.

[9] H. Busemann and B. B. Phadke, Locally desarguesian spaces, J. Geometry, 14 (1980), 123-138.

[10] _ Symmetric Spaces and Ellipses, Proc. Int. Christoffel Symposium, A collection of articles dedicated to E. B. Christoffel on the occasion of his 150th birthday, (1981), Birkhäuser, Basel, 626-635.

[11] _ Two theorems on general symmetric spaces, Pacific J. Math., 92 (1981), 39-48.

[12] _ Nonconvex spheres in G-spaces, J. Indian Math. Soc., 44 (1980), 39-50.

[13] D. Hilbert, Grundlagen der Geometrie, 10. Auflage, (1968), B. G. Teubner, Stuttgart.

[14] W. Hurewicz and H. Wallman, Dimension Theory, (1941), Princeton University Press, Princeton.

[15] P. J. Kelly and L. J. Paige, Symmetric perpendicularity in Hilbert geometries, Pacific J. Math., 2 (1952), 319-322.

[16] C. M. Petty, On the geometry of the Minkowski plane, Rivista di Matematica della Universita di Parma, 6 (1955), 269-292.

[17] A. V. Pogorelov, Hilbert's Fourth Problem, (Russian), (1974), Izdat. "Nauka", Moscow. The English translation (same title, (1979), V. H. Winston \& Sons, Washington D.C.) contains, in contrast to the original, a complete bibliography and 4 pages of useful notes. 
[18] L. S. Pontrjagin, Topological Groups, (1939), Princeton University Press, Princeton.

Received March 2, 1983.

THE UNIVERSITY OF SOUTHERN CALIFORNIA

LOS ANGELES, CA 90007

AND

The Fuinders University of South Australia

BEDFord PARK, Australia 



\title{
PACIFIC JOURNAL OF MATHEMATICS EDITORS
}

\author{
Donald BABBITt (Managing Editor) \\ University of California \\ Los Angeles, CA 90024 \\ J. DugunduI \\ University of Southern California \\ Los Angeles, CA 90089-1113 \\ R. FINN \\ Stanford University \\ Stanford, CA 94305 \\ HermanN FlaschKa \\ University of Arizona \\ Tucson, AŻ 85721
}

C. C. Moore

University of California

Berkeley, CA 94720

ARTHUR Ogus

University of California

Berkeley, CA 94720

Hugo Rossi

University of Utah

Salt Lake City, UT 84112

H. SAMELSON

Stanford University

Stanford, CA 94305

ASSOCIATE EDITORS
R. ARENS
E. F. BECKENBACH
B. H. NEUMANN
F. WOLF
K. YoSHIDA (1906-1982)

\section{SUPPORTING INSTITUTIONS}

$\begin{array}{ll}\text { UNIVERSITY OF ARIZONA } & \text { UNIVERSITY OF OREGON } \\ \text { UNIVERSITY OF BRITISH COLUMBIA } & \text { UNIVERSITY OF SOUTHERN CALIFORNIA } \\ \text { CALIFORNIA INSTITUTE OF TECHNOLOGY } & \text { STANFORD UNIVERSITY } \\ \text { UNIVERSITY OF CALIFORNIA } & \text { UNIVERSITY OF HAWAII } \\ \text { MONTANA STATE UNIVERSITY } & \text { UNIVERSITY OF TOKYO } \\ \text { UNIVERSITY OF NEVADA, RENO } & \text { UNIVERSITY OF UTAH } \\ \text { NEW MEXICO STATE UNIVERSITY } & \text { WASHINGTON STATE UNIVERSITY } \\ \text { OREGON STATE UNIVERSITY } & \text { UNIVERSITY OF WASHINGTON }\end{array}$

The Supporting Institutions listed above contribute to the cost of publication of this Journal, but they are not owners or publishers and have no responsibility for its content or policies.

Mathematical papers intended for publication in the Pacific Journal of Mathematics should be in typed form or offset-reproduced (not dittoed), double spaced with large margins. Please do not use built up fractions in the text of the manuscript. However, you may use them in the displayed equations. Underline Greek letters in red, German in green, and script in blue. The first paragraph must be capable of being used separately as a synopsis of the entire paper. In particular it should contain no bibliographic references. Please propose a heading for the odd numbered pages of less than 35 characters. Manuscripts, in triplicate, may be sent to any one of the editors. Please classify according to the scheme of Math. Reviews, Index to Vol. 39. Supply name and address of author to whom proofs should be sent. All other communications should be addressed to the managing editor, or Elaine Barth, University of California, Los Angeles, California 90024.

There are page-charges associated with articles appearing in the Pacific Journal of Mathematics. These charges are expected to be paid by the author's University, Government Agency or Company. If the author or authors do not have access to such Institutional support these charges are waived. Single authors will receive 50 free reprints; joint authors will receive a total of 100 free reprints. Additional copies may be obtained at cost in multiples of 50 .

The Pacific Journal of Mathematics is issued monthly as of January 1966. Regular subscription rate: $\$ 190.00$ a year (5 Vols., 10 issues). Special rate: $\$ 66.00$ a year to individual members of supporting institutions.

Subscriptions, orders for numbers issued in the last three calendar years, and changes of address should be sent to Pacific Journal of Mathematics, P.O. Box 969, Carmel Valley, CA 93924, U.S.A. Old back numbers obtainable from Kraus Periodicals Co., Route 100, Millwood, NY 10546.

The Pacific Journal of Mathematics at P.O. Box 969, Carmel Valley, CA 93924 (ISSN 0030-8730) publishes 5 volumes per year. Application to mail at Second-class postage rates is pending at Carmel Valley, California, and additional mailing offices. Postmaster: Send address changes to Pacific Journal of Mathematics, P.O. Box 969, Carmel Valley, CA 93924.

PUBLISHED BY PACIFIC JOURNAL OF MATHEMATICS, A NON-PROFIT CORPORATION

Copyright $@ 1984$ by Pacific Journal of Mathematics 


\section{Pacific Journal of Mathematics}

\section{Vol. 115, No. $2 \quad$ October, 1984}

Ersan Akyildiz, Gysin homomorphism and Schubert calculus ...........257

Marilyn Breen, Clear visibility and unions of two starshaped sets in the

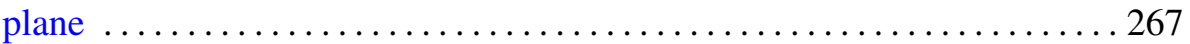

Robert F. Brown, Retraction methods in Nielsen fixed point theory . ......277

Herbert Busemann and Bhalchandra B. Phadke, A general version of Beltrami's theorem in the large ............................... 299

Gerald Arthur Edgar and Robert Francis Wheeler, Topological properties of Banach spaces ............................... 317

Yaakov Friedman and Bernard Russo, Conditional expectation without

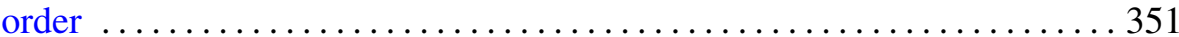

Robert Allen Goggins, Cobordism of manifolds with strong almost tangent

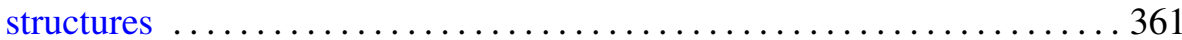

Mike Hoffman, Noncoincidence index of manifolds . . . . . . . . . . . . . 373

William H. Julian, $\varepsilon$-continuity and monotone operations $\ldots \ldots \ldots \ldots 385$

Gerasimos E. Ladas, Y. G. Sficas and I. P. Stavroulakis, Nonoscillatory

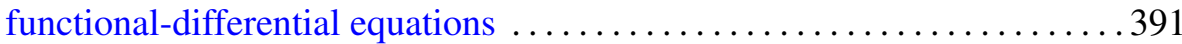

Arnold William Miller and Karel Libor Prikry, When the continuum has

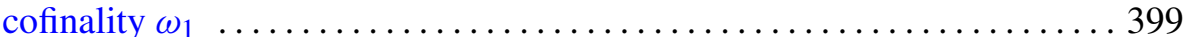

Jean-Leah Mohrherr, Density of a final segment of the truth-table

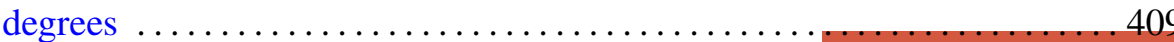

Carl Norman Mutchler, The flat Cauchy problem for radially hyperbolic operators from a characteristic manifold of high codimension ...

Kenji Nakagawa, On the orders of automorphisms of a closed Riemann surface

W. Ricker, Representation of vector-valued functions by Laplace transforms

Jorge Donato Samur, On semigroups of convolution operators in Hilbert

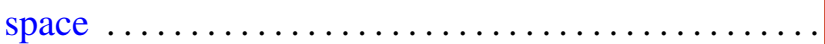

Joseph Gail Stampfli, One-dimensional perturbations of operators 481 Andrew George Earnest and John Sollion Hsia, Correction to: "Spinor norms of local integral rotations. II" 